\title{
Signal Non-Linearities And Their Effect Upon The Data Recovery Process In High-Density Optical Storage
}

\author{
P W Nutter", C D Wright*, I Gonzalez-Arcelus*, S D Jepson ${ }^{\#}$ \\ ${ }^{\#}$ Department of Computer Science, University of Manchester, Manchester, M13 9PL, UK \\ * School of Engineering \& Computer Science, University of Exeter, Exeter, EX4 4QF, UK
}

To predict the performance of optical read channels readout waveforms generated using linear superposition techniques are often used. The readout waveform is, however, often degraded by non-linear components particularly at high storage densities. In this paper the origins of such non-linearities are explained and their effects on read channel performance investigated. It is found that channel bit error rate can be properly optimised only if the signal non-linearity is taken into account.

Key words: optical storage channel, partial response maximum likelihood, PRML, diffraction analysis, superposition, inter-symbol-interference, ISI, non-linear.

\section{Introduction}

Diffraction analysis has not only proved to be an invaluable tool for the investigation of optical readout techniques, but also as an aid in the analysis and design of the read channel ${ }^{1)}$. However, the use of diffraction analysis is often restricted to the simulation of readout waveforms from short sequences of recorded data patterns; this is due to the associated high computational overheads and slow simulation speeds. When designing the read channel, the readout waveform due to a long data sequence is required ( $>10^{6}$ channel bits) in order to obtain a true measure of the channel bit-error-rate (BER) performance. In such applications, the readout signal is usually modelled by the linear superposition of system step or pulse responses. Such techniques permit the rapid generation of relatively accurate waveforms.

In high-density optical storage systems, such as the current DVD format and future short-wavelength derivatives, noticeable signal degradations exist in the readout waveform that are non-linear in nature and originate from (non-linear) inter-symbol-interference (ISI) between neighbouring recorded marks and cross-talk between adjacent tracks ${ }^{2), 3)}$. Modelling techniques based on the superposition of system step or impulse responses fail to take into account these signal degradations, due to their non-linear nature. As a result, the simulated waveforms fail to predict the true nature of the readout signal.

The aim of this paper is to investigate the effects of non-linear signal degradations on the performance of read channels designed and optimised using readout waveforms generated by linear superposition techniques.

\section{Origins and Effects of Non-Linear Readout Signal Components}

In order to improve the accuracy of linear superposed waveforms, whilst at the same time ensuring high computational speed, researchers have adopted a number of analytical techniques based upon curve fitting and optimisation processes, such as Volterra series ${ }^{4)}$. We, for example, have used a simple empirically derived technique based on signal amplitude adjustments during the linear (step) superposition process. Here, the linear superposition algorithm is modified to include an amplitude modification parameter, $\alpha_{k}$, i.e.

$$
r(t)=\sum_{k=-\infty}^{\infty} \mid c_{k} \alpha_{k} k\left(c_{k} t-k T_{b}\right)
$$

where $r$ is the readout waveform, $u(t)$ is the system step response, generated using a diffraction readout model, $c_{k}$ is the transition sequence $\in\{-1,0,1\}$ and $T_{b}$ is the channel bit length. Setting $\alpha_{k}=1$ for all $k$ results in the linear superposition of step responses. In order to simulate a more accurate (non-linear) waveform, values of $\alpha_{k}(\leq 1)$ are determined for each $k$, by using a least squares fit to minimise the MSE (mean-square-error) between the output waveform from the non-linear superposition algorithm and the 'true' readout waveform generated using a full diffraction simulation. For example, in the DVDROM system, the $3 \mathrm{~T}, 4 \mathrm{~T}$ and $5 \mathrm{~T}$ mark lengths contribute most significantly to the non-linear signal component ${ }^{3)}$. In this case, optimum values of $\alpha_{k}$ are 0.938 , 0.988 and 0.996 for $k=3 \mathrm{~T}, 4 \mathrm{~T}$ and $5 \mathrm{~T}$ respectively, and 1 elsewhere.

Although this simple technique permits the rapid generation of accurate readout waveforms, it requires an optimisation process over a large data sequence $\left(>1000 T_{b}\right)$. More recently we have developed a new approach, whereby the readout waveform is generated by the superposition of isolated pulse responses and non-linear signal components ${ }^{3}$.

The origin of the non-linear contributions can be explained by considering the case illustrated in Fig. 1, where the focused optical spot overlaps two neighbouring recorded marks on a disk. Assuming that the interaction of the optical field with the disk, and subsequent propagation to the collector lens is a linear process, then using the Babinet principle we can decompose the reflected light into isolated components due to non-overlapping features on the disk ${ }^{3), 5)}$ 


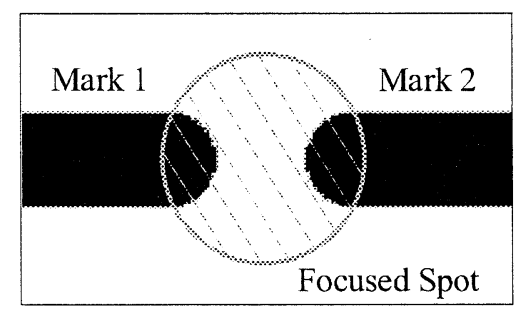

Fig. 1 Example disk structure

Thus, for the two marks in Fig. 1, the total reflected field from the disk, $\psi_{T}$, is given by the sum of isolated field components originating from the isolated features on the disk, i.e. the background, $\psi_{B}$, and the recorded marks, $\psi_{M I}$ and $\psi_{M 2}$, viz.

$$
\psi_{T}=\psi_{B}+\psi_{M 1}+\psi_{M 2}
$$

(co-ordinate indices have been removed for simplicity).

The field components propagate independently to the collector lens where they are collimated and recombined to give the total field distribution incident on the photodetector,

$$
\psi_{C}=p_{C} \cdot\left(\psi_{B}{ }^{\prime}+\psi_{M 1}{ }^{\prime}+\psi_{M 2}{ }^{\prime}\right)
$$

where $\psi_{B}{ }^{\prime}, \psi_{M I}{ }^{\prime}$ and $\psi_{M 2}{ }^{\prime}$ are the Fourier transforms of $\psi_{B}$, $\psi_{M 1}$ and $\psi_{M 2}$ respectively, and $p_{C}$ is the collector lens aperture pupil function.

The total signal from the photodetector is given by

$$
I\left(x_{s}\right)=\iint_{-\infty}^{\infty}\left|p_{C}\right|^{2}\left|\psi_{B}+\psi_{M 1}+\psi_{M 2}\right|^{2} d x_{2} d y_{2}
$$

and expanding and separating components gives

$$
\begin{aligned}
I\left(x_{s}\right)= & \text { linear term in intensity }+ \\
& \iint_{-\infty}^{\infty}\left|p_{C}\right|^{2}\left\{2\left|\psi_{M 1} \| \psi_{M 2}\right| \cos \Delta \phi_{M 1 M 2}\right\} d x_{2} d y_{2}
\end{aligned}
$$

where $\Delta \phi_{M 1 M 2}$ is the phase difference between the field components due to the two isolated marks. The linear term in intensity represents the signal produced using linear superposition of isolated systems step responses. The integral in Eqn (5) represents a signal component, linear in amplitude and not intensity, that is due to the correlation of the two field components generated from the isolated marks; this signal component constitutes non-linear ISI in the readout waveform.

The error in a linearly superposed waveform can be determined by measuring the difference between waveforms generated using linear (step) superposition and diffraction analysis. Fig. 2 illustrates the error generated for the simple case of Fig. 1; also plotted is the non-linear signal contribution calculated from Eqn (5). It is clear that the error in the linearly superposed waveform is due entirely to the absence of the non-linear contributions described by Eqn(5). In light of this, we have developed a new superposition approach, termed 'pulse superposition with non-linearity correction', that includes both linear and non-linear contributions to the readout signal. The simulation process is explained in detail elsewhere ${ }^{3)}$ and described briefly below.

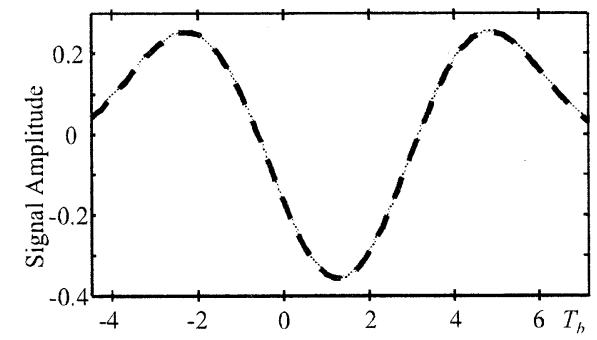

Fig. 2 Comparison of the calculated error in the superposition process (solid line) and the non-linear ISI component of Eqn (5) for the case of Fig. 1.

First, a family of isolated pulse responses is generated using a full diffraction model, for each mark length allowed by the run-length-limited (RLL) coding scheme used by the channel. These pulse responses are then superposed, according to the supplied RLL data, to form a linear readout waveform. Next, a family of non-linear ISI signal components is generated for each mark separation allowed by the RLL coding scheme. This follows the analysis presented in the previous section, where the signal components are generated using neighbouring mark lengths equal to the average run-length of the coding scheme. These signal components are then superposed, according to the supplied RLL data, to generate a non-linear waveform representing the signal contributions due to non-linear ISI. Adding the linear and non-linear signal contributions produces the final 'corrected' superposed waveform.

Fig. 3 illustrates a sample of waveforms generated using full diffraction analysis, step superposition (linear waveform) and the new non-linearity correction technique, for the minimum $3 T_{b}$ mark spacing in the DVDROM system (where the dominant non-linear ISI component occurs). The effect of the non-linearity inherent in the readout process itself is clear. Simple linear superposition (of step responses) is a relatively poor fit to the true readout signal, particularly for closely spaced marks. Using the new technique the accuracy of the simulation is improved significantly. The MSE between a diffraction waveform and the non-linear waveform (generated using the proposed technique) is $-66 \mathrm{~dB}$, compared with $-31 \mathrm{~dB}$ for the linear (step superposed) waveform.

\section{Read Channel Modelling and Design}

The fact that the readout signal is in essence non-linear means that linear methods used to design the data recovery channel may result in a non-optimal design. In order to investigate the performance of read channels, we have used an extensive channel simulation that allows us to predict the bit-error-rate (BER) performance of threshold and PRML channels ${ }^{1)}$. Here we concentrate on PRML channels, as illustrated in Fig. 4.

The RLL encoded data, $a_{k}$, is used to generate the readout waveform, $r(t)$, with noise contributions due to media noise, $n_{m}(t)$, and electronic noise, $n_{e}(t)$, added. The readout 
waveform is filtered to remove high-frequency noise by a continuous time filter (CTF) and sampled at a data rate of $1 / T_{b}$. The sampled data is equalised to a desired PR target using an FIR digital filter. The filter tap coefficients are designed to minimise the MSE between the output of the FIR filter and the ideal PR output, using the least mean square (LMS) algorithm ${ }^{6}$. Finally the data is recovered using a Viterbi detector designed for the PR polynomial and RLL code being used and having a maximum of $2^{v}$ states, where $v$ is the order of the PR polynomial ${ }^{7)}$.

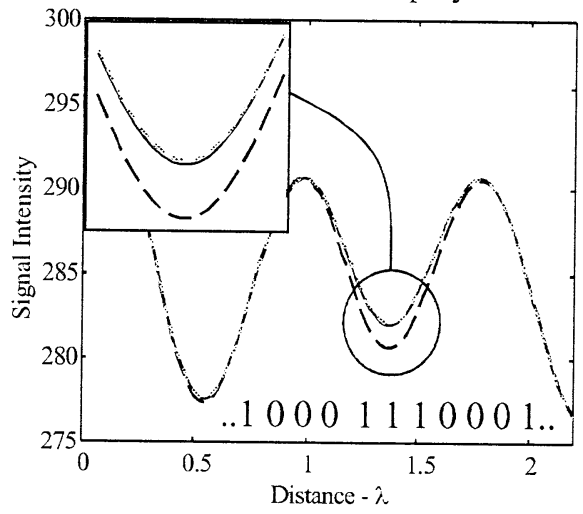

Fig. 3 Comparison of readout waveforms: diffraction analysis (solid line), step superposition (dashed line) and pulse superposition with non-linearity correction (grey dashed line).

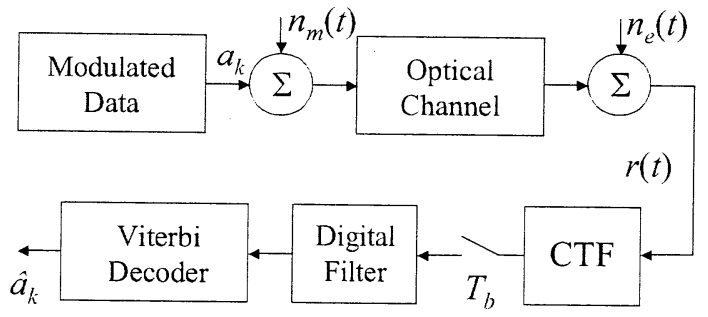

Fig. 4 The PRML optical recording channel

Error events in the Viterbi trellis occur if the estimated state sequence and the correct state sequence are equal at times $k_{l}$ and $k_{2}$ but nowhere in between. If the length of the event is defined as $m=k_{2}-k_{1}-1$, the detector will choose the incorrect sequence, $\hat{a}_{k}$, over the correct sequence, $a_{k}$, if

$$
\sum_{k=k_{1}}^{k_{1}+m}\left(\left(\tilde{y}_{k}+n_{k}\right)-\hat{y}_{k}\right)^{2}<\sum_{k=k_{1}}^{k_{1}-m}\left(\left(\widetilde{y}_{k}+n_{k}\right)-y_{k}\right)^{2}
$$

where $\tilde{y}_{k}$ is the equalized readout signal, $n_{k}$ is the noise component, and $y_{k}$ is the ideal noiseless output.

When perfect equalization is achieved and the noise is additive white Gaussian noise (AWGN), the probability of error is dominated by minimum distance error events and a purely analytical solution for BER can be given approximately as

$$
P_{h} \approx K Q\left(\frac{d_{\min }}{2 \sigma}\right)
$$

where $\sigma$ is the noise standard deviation at the input to the detector, $K$ is a constant, the function $Q(\sim)$ is defined as

$$
Q(x)=\frac{1}{\sqrt{2 \pi}} \int_{x}^{\infty} e^{-z^{2} / 2} d z
$$

and $d_{\min }$ is the minimum Euclidean distance of all allowable output error sequences, $e_{k},{ }^{7)}$

$$
e_{k}=\bar{y}_{k}-y_{k} \text {. }
$$

When the readout signal is not equalized exactly to the PR target response or the noise is coloured, then the probability of an error event is data dependent. Defining $i=k-k_{l}$, an error at the detector will occur if

$$
\sum_{i=0}^{m}\left(\tilde{y}_{i}-\hat{y}_{i}+n_{i}\right)^{2}<\sum_{i=0}^{m}\left(\tilde{y}_{i}-y_{i}+n_{i}\right)^{2} .
$$

Using Eqn(9) and rearranging we have

$$
\sum_{i=0}^{m} \frac{1}{2}\left\{e_{i}^{2}-2 \cdot e_{i} \cdot\left(\tilde{y}_{i}-y_{i}\right)\right\}<\sum_{i=0}^{m} e_{i} \cdot n_{i}
$$

The right-hand side of Eqn(11) is a Gaussian random variable. Therefore, the probability of the detector choosing the incorrect sequence, $\hat{a}_{k}$, over the correct sequence, $a_{k}$, is given by

$$
\operatorname{Pr}(a \rightarrow \hat{a})=Q\left(\frac{\sum_{i=0}^{m}\left(e_{i}^{2}-2 \cdot e_{i} \cdot\left(\tilde{y}_{i}-y_{i}\right)\right)}{2 \sqrt{\sum_{p=0}^{m} \sum_{q}^{m} e_{p} e_{p} R(p-q)}}\right)
$$

where $R$ is the autocorrelation of the noise at the input to the detector. In order to estimate the BER we must average over all possible error events.

The model simulates the signal generation and signal processing stages of the channel numerically, while using the analytical description, in Eqn (12), of the error events to calculate a BER (a full numerical simulation in which actual errors are counted individually can be performed if desired, but takes considerably longer). Error event probability is calculated for all possible events of given lengths. The lengths tested are chosen depending upon the PR scheme and modulation code being used. Each error event contains a correct recorded data sequence, $a_{k}$, that meets the modulation code constraints, and an incorrect sequence, $\hat{a}_{k}$, that meets the detector code constraints.

The error event probability is weighted by the number of bit errors contained in the event, $w_{H}$, and the probability of the correct sequence occurring, $\operatorname{Pr}(a)$, and is summed over all possible sequence pairs to give an estimate of the BER

$$
P_{h} \approx \sum_{\text {allaallâa }} \sum_{\operatorname{ar}} \operatorname{Pr}(a) w_{H} \operatorname{Pr}(a \rightarrow \hat{a}) .
$$

\section{Results}

Using the non-linear superposition technique discussed in $\$ 2$, we have investigated how the performance of PRML read channels, designed and optimised using linear (step superposed) waveforms, are affected by non-linear signal degradations originating from ISI.

The DVDROM system was investigated, assuming a channel bit size of $0.133 \mu \mathrm{m}$ and $\operatorname{RLL}(2,10)$ encoding. It is assumed that the noise contributions arise from $50 \%$ media noise, coloured by the channel, and $50 \%$ electronic, white 
noise. A 15-tap FIR filter was used to equalise the readout waveform to match a desired PR target. A number of PR targets were investigated, all of which exhibit a frequency characteristic close to that of the optical channel.

Fig. 5 illustrates the BER performance v. SNR of the implemented PRML read channels, with the wideband SNR defined as

$$
S N R_{W B}=10 \log _{10}\left(\overline{s^{2}} / N_{B}\right)
$$

where $\overline{s^{2}}$ is the mean-square value of the signal (i.e the total signal power) and $N_{B}$ is the total integrated noise power (in this case over a bandwidth of $20 \mathrm{MHz}$ ). Two cases are shown, 1) the predicted performance using linear waveforms and 2) the predicted performance using non-linear waveforms (generated using the new technique). For each particular PRML scheme the same FIR filter was used for both linear and non-linear signals, the taps having been determined using linear waveforms (and the same tap values were used at each SNR). It is evident that PRML read channels designed using linear waveforms exhibit acceptable BER performance, even when exposed to 'true' non-linear readout waveforms. Indeed, the BER is lower when the 'true' waveform is processed by a channel previously optimised using linearly superposed signals. The conventional design approach has therefore underestimated the likely BER performance.
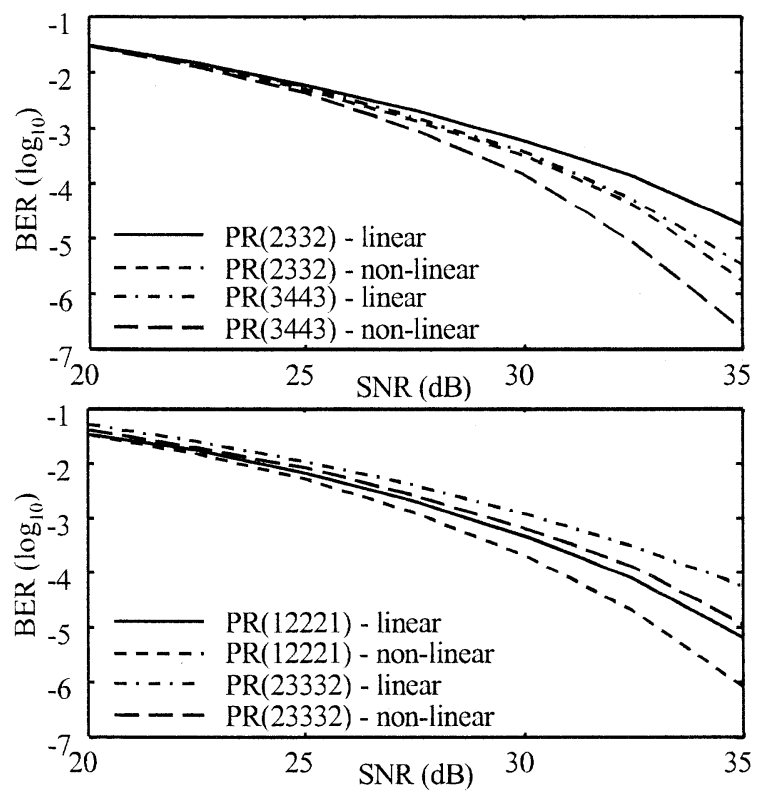

Fig. 5 BER curves for PR schemes with FIR taps optimised using linear waveforms.

Of the four PRML schemes investigated above, the PR(3443) channel performs best in terms of BER, and we chose this scheme for further investigation. In particular it is important to know if further improvements in BER performance can be achieved by proper optimisation of the read channel. Results are shown in Fig. 6. Three conditions are illustrated, all for a PR(3443) channel: the solid line shows the performance of the channel when the input signal is linear and the FIR taps are designed for a linear signal; the dotted line shows the performance when the input signal contains the non-linear components but the taps are designed assuming a linear signal; and the dashed line is for the case of a non-linear input signal with FIR taps optimised for that non-linear waveform. It is clear that 'correct' optimisation of the FIR taps when using the non-linear input waveform produces the best (lowest BER) performance. This is of practical importance, since in reality readout waveforms will contain the non-linear components that we have described.

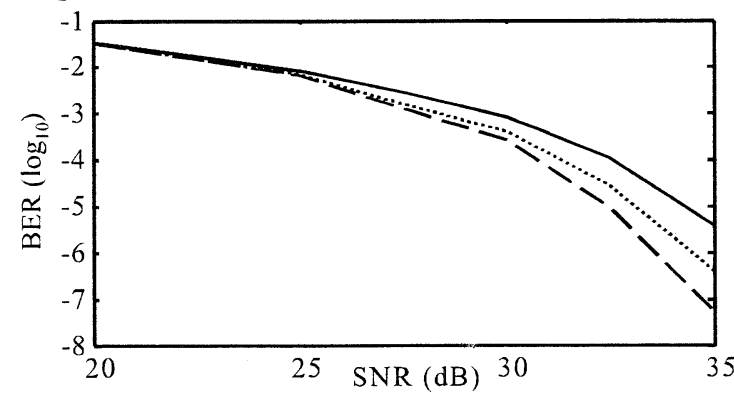

Fig. 6 BER curves: channel optimised and simulated using linear waveforms (solid line), channel optimised using linear waveforms, simulated using non-linear waveforms (small dashed line), and channel optimised and simulated using non-linear waveforms (dashed line)

\section{Conclusions}

A fast and accurate method for including the effects of readout non-linearities on channel performance in high-density optical storage formats has been presented. Although PRML read channels optimised using linear waveforms produce acceptable results, significant improvements in BER performance can be achieved if non-linear effects are accounted for properly. In the case of the PR(3443) read channel analysed, for a BER of $10^{-5}$, a SNR gain of $2 \mathrm{~dB}$ is observed compared to that predicted using linear signal modelling techniques. Further work will compare these theoretical results with experimental analysis.

\section{References}

1) C.D.Wright, P.W.Nutter, M.K.Loze and S.D. Jepson: IEEE Trans Cons Elec, 46, p.586, (2000).

2) H.Cho, J.An, S.Ong, D.Hong: Opt. Eng., 40 (8), p.1621 (2001).

3) P.W.Nutter and C.D.Wright: Proc. SPIE, 4342, p.364 (2001)

4) L.Agarossi, S.Bellini, A.Canella and P.Migliorati: Proc. IEEE Int. Conf. Acoustics, Speech \& Signal, 3, p.1605 (1998).

5) T.D.Milster: Appl. Opt., 37 (29), p.6878 (1998).

6) I.Ozgunes and B.V.K.Kumar: Proc. SPIE, 2605, p.77 (1995).

7) G.D.Fonney: IEEE Trans, IT-18, p.363 (1972).

Received May 28, 2002; Accepted August 9, 2002 